\title{
All-organic dielectric-percolative three-component composite materials with high electromechanical response
}

\author{
Cheng Huang and Q. M. Zhang ${ }^{\text {a) }}$ \\ Materials Research Institute and Electrical Engineering Department, The Pennsylvania State University, \\ University Park, Pennsylvania 16802 \\ Gal deBotton and Kaushik Bhattacharya \\ Division of Engineering and Applied Science, California Institute of Technology, Pasadena, California 91125
}

(Received 8 January 2004; accepted 1 April 2004; published online 12 May 2004)

\begin{abstract}
By combining the high-dielectric copper phthalocyanine oligomer (PolyCuPc) and conductive polyanline (PANI) within polyurethane (PU) matrix an all-organic three-component dielectric-percolative composite with high dielectric constant is demonstrated. In this three-component composite system, the high-dielectric-constant PolyCuPc particulates enhance the dielectric constant of the PU matrix and this combined two-component dielectric matrix in turn serves as the high-dielectric-constant host for the PANI to realize percolative phenomenon and further enhance the dielectric response. As a result, an electromechanical strain of $9.3 \%$ and elastic energy density of $0.4 \mathrm{~J} / \mathrm{cm}^{3}$ under an electric field of $20 \mathrm{~V} / \mu \mathrm{m}$ can be induced. (C) 2004 American Institute of Physics. [DOI: 10.1063/1.1757632]
\end{abstract}

Electroactive polymers (EAPs) are capable of converting energy in the form of electric charge and voltage to mechanical force and movement and vice versa. In recent years, several electroactive polymer actuator materials, whose responses are controlled by external electric fields, have generated considerable interest. ${ }^{1-4}$ However, one severe drawback of these EAPs is the very high operation electric field $(>100 \mathrm{~V} / \mu \mathrm{m})$ required to generate a high strain with elastic energy density much higher than those in the current available piezoelectric materials (elastic energy density $>0.1$ $\left.\mathrm{J} / \mathrm{cm}^{3}\right){ }^{5,6}$ As has been pointed out in earlier publications, ${ }^{7,8}$ one fundamental cause for such high fields is the low dielectric constant of these EAPs. From the energy conservation, the elastic energy density generated in an electroactive polymer cannot exceed the input electric energy density, which is $K \epsilon_{0} E^{2} / 2$ for a linear dielectric, where $K$ is the dielectric constant, $\epsilon_{0}=8.85 \times 10^{-12} \mathrm{~F} / \mathrm{m}$, and $E$ is the applied field. This simple fact points out the importance of raising the dielectric constant of the EAPs to achieve high electromechanical response with low applied fields.

Recently high dielectric and electromechanical responses have been demonstrated in two-component composite actuator materials. ${ }^{7-9}$ Especially, a two-component percolative composite, fabricated by a combination of conductive polymer particulates [polyaniline (PANI)] within the relaxor ferroelectric polymer matrix, raises the dielectric constant $(>1000)$ substantially higher than the polymer matrix $(\sim 50){ }^{8,10,11}$ In composites of conductive fillers embedded in an insulation matrix, the variation of the dielectric constant of the composite with the concentration $f$ of the conductive filler has been predicted to follow a critical behavior, ${ }^{12,13}$

$$
K_{c}=K_{m}\left(\frac{f_{c}-f}{f}\right)^{-q},
$$

${ }^{a)}$ Electronic mail: qxz1@psu.edu where $K_{m}$ is the dielectric constant of the matrix, $q$ is a critical exponent, and $f_{c}$ is the percolation threshold for the conduction. As shown in Eq. (1), a high matrix dielectric constant $K_{m}$ is a necessary condition to achieve very high dielectric constant for the percolative composites even when the conductive filler concentration $f$ is not very close to $f_{c}$. For example, for the two-component percolative composites previously investigated, the $\mathrm{P}(\mathrm{VDF}-\mathrm{TrFE}-\mathrm{CTFE})$ terpolymer was used as the polymer matrix, which possesses a relatively high room-temperature dielectric constant $(\sim 50){ }^{8}$ Unfortunately, most of the conventional dielectric polymers and elastomers have a low dielectric constant $(<10)$. This means that if they are used as the matrix, the volume fraction of the conductive polymer must be very close to the percolation threshold, $f_{c}$, in order to reach the high dielectric constant. However, as $f$ approaches $f_{c}$, the composites will become very sensitive to the composition variation and the breakdown field will also become low, both of which are not desirable for the electromechanical applications of the composites.

To overcome these drawbacks, we propose a different strategy to raise the dielectric constant of the polymer matrix. A two-component dielectric composite is used as the matrix for the percolative composite, which consists of the PolyCuPc oligomer (dielectric constant $K>10^{6}$ ) guest embedded in a polymer matrix host. ${ }^{14}$ The resulting threecomponent composites with the conductive polymer fillers in the dielectric composite host can be driven with a relatively high breakdown field $(>20 \mathrm{~V} / \mu \mathrm{m})$, and exhibit significantly enhanced dielectric and electromechanical responses. The advantage of this approach is the matrix in the twocomponent dielectric composite can be chosen from a wide range of polymers. ${ }^{15}$

The PolyCuPc oligomer was synthesized by the solution method with a minor modification. The elastic modulus of PolyCuPc is $2 \mathrm{GPa}$ at room temperature. ${ }^{16}$ PANI was synthesized using the conventional polymerization of aniline to 

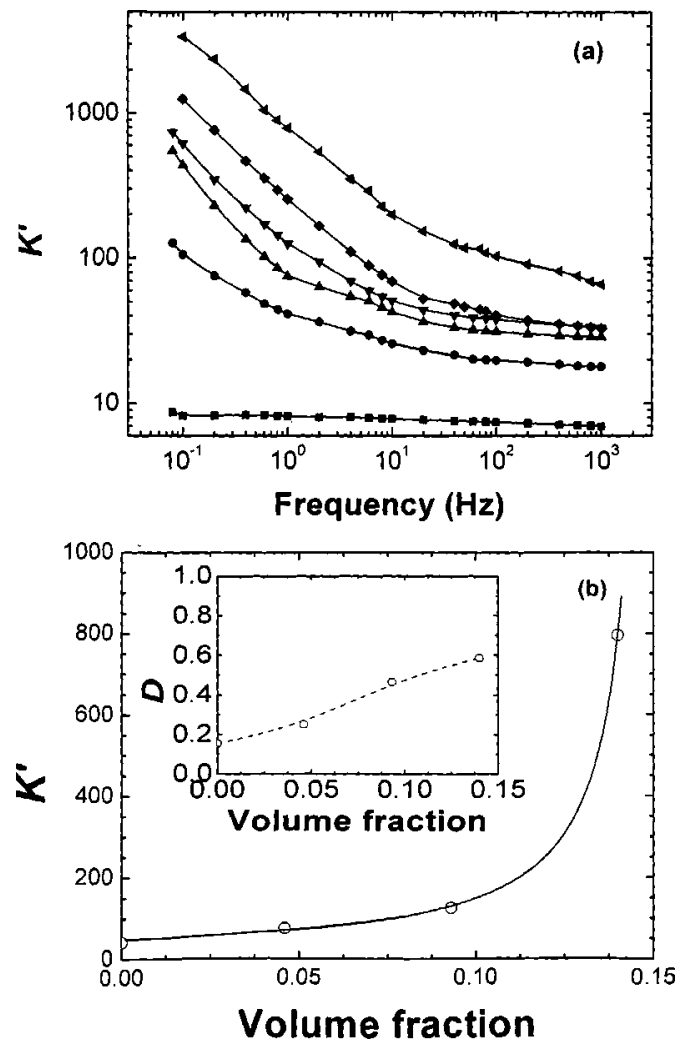

FIG. 1. (a) Dielectric constant $K^{\prime}$ as a function of frequency measured at room temperature for the composites with different volume fraction of the PolyCuPc and PANI. Curves from the bottom to top are $(x \mathrm{PANI} / y \mathrm{PolyCuPc} /(1-y) \mathrm{PU})$ : PU matrix, $0 / 15 / 85,4.6 / 15 / 85,9.3 / 15 / 85$, $0 / 30 / 70$, and $14 / 15 / 85$. The data points are shown and solid curves are drawn to guide eyes. (b) Evolution of $K^{\prime}$ vs the PANI volume fraction, (15PolyCuPc/85PU as the dielectric matrix), measured at room temperature and at $1 \mathrm{~Hz}$. The continuous line represents the fitting to Eq. (1). Inset shows the dielectric loss of the composites vs the PANI volume fraction.

produce fine emeraldine hydrochloride conductive polymer particulates. ${ }^{17}$ The PANI powder has an elastic modulus of $2.3 \mathrm{GPa}$ at room temperature. ${ }^{17,18}$ The PU matrix is Dow 2103-80AE, which has an elastic modulus of $28 \mathrm{MPa}$. Composite films of PolyCuPc and PANI within PU matrix were prepared using the solution cast and then hot press method. PU powder was dissolved in dimethylformamide and then a proper amount of PolyCuPc and PANI powders were added to the solution. After that, the solution was poured onto a glass plate and dried. The typical film thickness was about 50 $\mu \mathrm{m}$, which can be controlled by the hot press of the solution cast composite films. To facilitate the discussion and comparison of the composites, the composition of the composites is labeled as $x \mathrm{PANI} / y \mathrm{PolyCuPc} /(100-y) \mathrm{PU}$, where the volume ratio of PolyCuPc/PU is $y /(100-y)$ and $x$ is the volume fraction of PANI in the composite.

The dielectric properties of the composites were characterized by measuring the magnitude and the phase of the current passing through the composite by a lock-in amplifier (SRS, Model SR830 DSP) under a given ac voltage to obtain the complex electric impedance $Z^{*}=1 /\left(j \omega C^{*}\right)$ from which both the real part $K^{\prime}$ of the dielectric constant and dielectric loss can be determined. The elastic modulus $Y$ and mechanical loss were determined using a commercial dynamic mechanical analyzer (TA Instruments, DMA2980). The electricfield-induced strain along the applied-field-direction Downloaded 12 Jan 2006 to 131.215.240.9. Redistribution subject
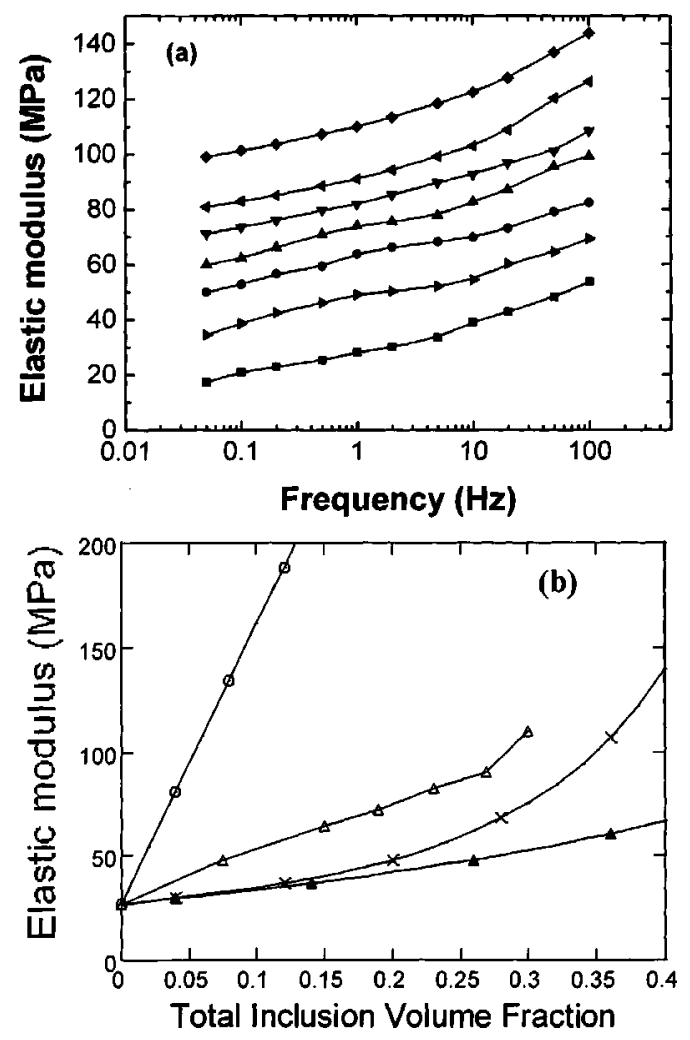

FIG. 2. (a) Elastic modulus vs frequency measured at room temperature for the composites. Curves from the bottom to top are $(x \mathrm{PANI} / y \mathrm{PolyCuPc} /(1$ $-y) \mathrm{PU})$ : PU matrix, 0/7.5/92.5, 0/15/85, 4.6/15/85, 9.3/15/85, 14/15/85, and $0 / 30 / 70$. The data points are shown and solid curves are drawn to guide eyes. (b) The elastic modulus measured at $1 \mathrm{~Hz}$ of the composites vs the total inclusion volume fraction (PolyCuPc and PANI) for the composites (open triangles). The solid line is drawn to guide eyes. And comparison with the model results for the composites (curves with open circles and closed triangles: HS upper and lower bounds; curve with crosses: SCS model result).

(longitudinal strain, $S_{3}$ ) was measured using a cantileverbased-sensor system. The strain signal was detected by a photonic sensor (MTI2000).

Presented in Fig. 1(a) is the dielectric constant $K^{\prime}$ for the composites with different volume fraction of the PANI as well as PolyCuPc as a function of frequency measured at room temperature. Compared with the pure PU matrix, which has a dielectric constant of 8 (at $1 \mathrm{~Hz})$, the dielectric constant was raised to 41 in the $15 \mathrm{PolyCuPc} / 85 \mathrm{PU}$ twocomponent composite and $K^{\prime}$ was further raised to 270 in $30 \mathrm{PolyCuPc} / 70 \mathrm{PU}$ composite. However, when using the two-component composite matrix 30PolyCuPc/70PC for the percolative composites, it was found that the elastic modulus of the three-component composite becomes quite high and breakdown field is low. Therefore, in this study, the twocomponent dielectric composite $15 \mathrm{PolyCuPc} / 85 \mathrm{PU}$ was employed as the dielectric matrix for the percolative composites. As can be seen, the three-component dielectricpercolative composite, 14PANI/15PolyCuPc/85PU, shows a high dielectric constant of 800 .

Summarizing the data in Fig. 1(a), the real part of dielectric constant at $1 \mathrm{~Hz}$ as a function of the volume fraction of PANI in the dielectric composite matrix of 15PolyCuPc/ 85PU, yields the data in Fig. 1(b). Fitting the data in Fig. (b) to Eq. (1) yields $f_{c}=0.15$, and $q=1.0$. The value of the critical exponent $q$ is close to $q=0.9$ predicted by the perco- 

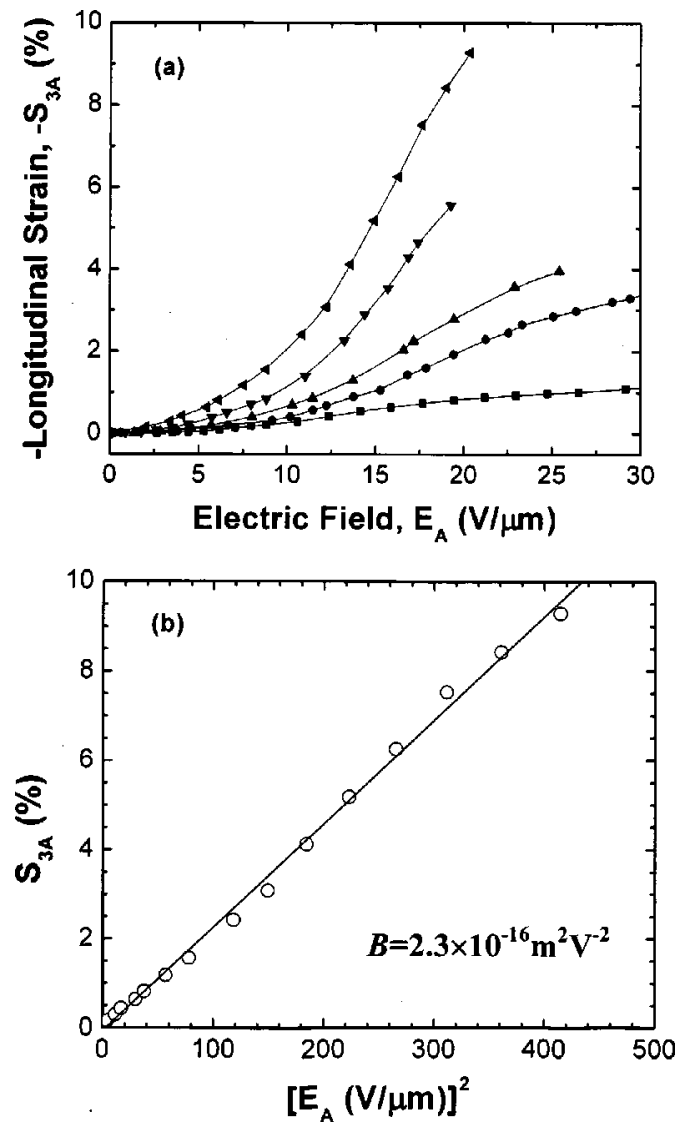

FIG. 3. (a) The thickness strain amplitude $S_{3 A}$ as a function of the appliedfield amplitude $E_{A}$ measured at room temperature for the composites. The applied-field frequency is $1 \mathrm{~Hz}$. Curves from the bottom to top are for the samples $(x \mathrm{PANI} / y$ PolyCuPc/( $1-y) \mathrm{PU})$ : PU matrix, 0/15/85, 4.6/15/85, $9.3 / 15 / 85$, and $14 / 15 / 85$. Data points are shown and curves are drawn to link the data points. (b) Variation of the strain amplitude $S_{3 A}$ with the square of the applied-field amplitude $E_{A}^{2}$ for the composite of 14PANI/15PolyCuPc/ $85 \mathrm{PU}$.

lation theory for a three-dimensional percolative system. ${ }^{12,13}$ In the inset of Fig. 1(b), the dielectric loss shows continuous increase with PANI concentration which seems to be inconsistent with the prediction of percolation theory. ${ }^{12,13}$ It can be shown that this is caused by the inhomogeneity in the composites. ${ }^{19}$ In a real composite, there exists small composition variation which can affect the imaginary part of the dielectric constant quite significantly. ${ }^{13,15,19}$

The elastic modulus and mechanical loss for these composites are presented in Fig. 2. Due to the high modulus of the PolyCuPc and the conductive polymer PANI in comparison with the PU matrix the elastic modulus of the composites increases with the filler concentration. To understand how the PolyCuPc and PANI fillers affect the overall elastic behavior of the composites, the measured effective elastic moduli are compared with two well-known models. As shown in Fig. 2(b), the experimental results lie in between the HS lower and upper bounds and seem to be more consistent with the SCS model result. ${ }^{20,21}$ We note, however, that the SCS model usually overestimates the modulus of two phase composites. Thus, the fact that the measured moduli are higher than the SCS estimates imply that the examined composite samples probably exhibit overall anisotropy in their elastic behavior.
The field induced strain response for the composite was characterized. Figure 3(a) presents the amplitude of the strain response $S_{3 A}$ as a function of the applied field amplitude $E_{A}$. Under a field of $20 \mathrm{~V} / \mu \mathrm{m}$, a high thickness strain of $-9.3 \%$ can be obtained for the three-component dielectricpercolative composite of $14 \mathrm{PANI} / 15 \mathrm{PolyCuPc} / 85 \mathrm{PU}$, while the pure PU has a $-0.82 \%$ strain and the two-component composite dielectric matrix, $15 \mathrm{PolyCuPc} / 85 \mathrm{PU}$, has a $-1.92 \%$ strain under the same field. Combining the strain and the elastic modulus (91 MPa) for the composite yields the elastic energy density of about $0.4 \mathrm{~J} / \mathrm{cm}^{3}$ for the threecomponent composite under an electric field of $20 \mathrm{~V} / \mu \mathrm{m}$. In comparison with the PU matrix, the three-component dielectric-percolative composite represents a ten times increase in the strain response and more than three hundred times increase in the elastic energy density. Compared with the dielectric elastomers reported earlier and the fluoropolymer based relaxor ferroelectric electrostrictive polymers, the results reported here represent one order of magnitude reduction in the applied field to achieve the similar strain and elastic energy density. Figure 3(b) shows the relationship between the strain amplitude $S_{3 A}$ and the square of the appliedfield amplitude $E_{A}^{2}$ for the sample of 14PANI/15PolyCuPc/ 85PU. The solid line in the figure is fitting of the data to equation $S=B E^{2}$ which yields an electric field induced strain coefficient $B=2.3 \times 10^{-16} \mathrm{~m}^{2} / \mathrm{V}^{2}$.

The authors gratefully acknowledge the financial support of this work by NIH under Grant No. 8R01EB002078-04 and Chinese National Natural Science Foundation under Contract No. 50228304.

${ }^{1}$ Electroactive Polymer (EAP) Actuators as Artificial Muscles-Reality, Potential, and Challenges, edited by Y. Bar-Cohen (SPIE, Bellingham, WA, 2001).

${ }^{2}$ S. Ashley, Sci. Am. 289, 52 (2003).

${ }^{3}$ F. Xia, T. B. Xu, S. Tadigadapa, and Q. M. Zhang, Proceedings of the Seventh International Conference on Micro Total Analysis Systems $(\mu-$ TAS), 2003, p. 1.

${ }^{4}$ T. B. Xu, Z.-Y. Cheng, and Q. M. Zhang, Appl. Phys. Lett. 80, 1082 (2002).

${ }^{5}$ Q. M. Zhang, V. Bharti, and X. Zhao, Science 280, 2101 (1998).

${ }^{6}$ R. Pelrine, R. Kornbluh, Q. Pei, and J. Joseph, Science 287, 836 (2000).

${ }^{7}$ Q. M. Zhang, H. Li, M. Poh, Z.-Y. Cheng, H. Xu, F. Xia, and C. Huang, Nature (London) 419, 284 (2002).

${ }^{8}$ C. Huang, Q. M. Zhang, and J. Su, Appl. Phys. Lett. 82, 3502 (2003)

${ }^{9}$ I. Vrejoiu, J. D. Pedarning, D. Bauerle, and M. Dinescu, Appl. Phys. Lett. 83, 2130 (2003).

${ }^{10}$ H. Xu, Z.-Y. Cheng, D. Olson, T. Mai, Q. M. Zhang, and G. J. Kavarnos, Appl. Phys. Lett. 78, 2360 (2001).

${ }^{11}$ F. Xia, Z.-Y. Cheng, H. Xu, H. Li, Q. M. Zhang, G. Kavarnos, R. Ting, G. Abdel-Sadek, and K. Belfield, Adv. Mater. (Weinheim, Ger.) 14, 1574 (2002).

${ }^{12}$ A. L. Efros and B. I. Shklovskii, Phys. Status Solidi B 76, 475 (1976).

${ }^{13}$ C.-W. Nan, Prog. Mater. Sci. 37, 1 (1993).

${ }^{14}$ C. Huang, R. Klein, F. Xia, H. Li, Q. M. Zhang, F. Bauer, and Z.-Y. Cheng, IEEE Trans. Dielectr. Electr. Insul. 11, 1 (2004).

${ }^{15}$ Z. M. Dang, Y. Shen, and C.-W. Nan, Appl. Phys. Lett. 81, 4814 (2002).

${ }^{16}$ B. N. Achar, G. Fohlen, and J. Parker, J. Polym. Sci., Polym. Chem. Ed. 20, 1785 (1982).

${ }^{17}$ J. Akhavan and K. Slack, Synth. Met. 124, 363 (2001).

${ }^{18}$ S. Hardaker and R. Gregory, Polymer Data Handbook, edited by J. Mark (Oxford University Press, New York, 1999), p. 271.

${ }^{19}$ C. Huang and Q. M. Zhang, Adv. Funct. Mater. (to be published).

${ }^{20}$ Z. Hashin and S. Shtrikman, J. Mech. Phys. Solids 10, 335 (1962).

${ }^{21}$ J. R. Willis, J. Mech. Phys. Solids 25, 185 (1977). 